Int J Infect Microbiol 2013;2(3):105-110

\section{Changing pattern of resistant pathogens causing urinary tract infections in Karachi}

\author{
Najmul H,* Tanveer A
}

Department of Microbiology, University of Karachi, Karachi 75720, Pakistan

*Correspondence to: Najmul Hasan, Department of Microbiology, University of Karachi, Karachi 75720, Pakistan, email: najam.edu.pak@gmail.com, Tel. No.: (+92)-3333669331
ABSTRACT

INTODUCTION: The study under view is based under the aim to investigate the prevalence and susceptibility pattern of pathogens, causing urinary tract infections (UTIs), to antibiotics commonly used in routine medication.

MATERIALS AND METHODS: Over a period of 10 months 100 isolates were collected for the determination of their susceptibility to chosen antibiotics, from a laboratory (MedPath Laboratories) in urban area of Karachi. All Gramnegative and Gram-positive urinary tract pathogens were re-identified by their morphological and biochemical characteristics and the susceptibility to seven antibiotics was determined.

RESULTS: Pathogens were found as, Escherichia coli, Pseudomona spp, Klebsiella species, Enterobacter spp., and Staphylococci spp. In recent study, more than half of the Escherichia coli isolates were resistant to one or more of the all antimicrobial drugs tested. Resistance was most common to amoxicillin/clavulanic acid and ofloxacin, cefixime, followed by gentamicin. Our results indicate that Escherichia coli and Pseudomonas spp. were the most common organisms causing UTI. Other organisms involved were Enterobacter spp., Staphylococcus spp., and Klebsiella spp. Increasing patterns of resistant to gentamicin, and ofloxacin were also observed.

CONCLUSIONS: In conclusion, pattern of antibiotic susceptibility to first line antibiotics is changing hence antimicrobial susceptibility testing of all isolates is crucial for the treatment of UTI.

KEY WORDS: Resistance, Antibiotics, Pathogens, Infections, Karachi

Article submitted 5 May. Reviewed 20 May. Author correction 20 August. Final version accepted 25 August 2013. 


\section{INTRODUCTION}

Although antimicrobial resistance with Streptococcus pneumoniae was first recognized in 1917. However in the late 1930s when Fleming described usage of penicillin, the beginning of the antibacterial era was heralded. ${ }^{1}$ Antibiotics have been widely used because of their wide spectrum of activity. For example the fluoroquinolones have been utilized to treat a great variety of infections. ${ }^{2} \mathrm{~A}$ few years ago, ciprofloxacin was pointed out as the most consumed antibacterial agent worldwide. ${ }^{2-3}$ This high level of use, and to some extent of abuse in the sense of gratuitous usage, or use of quinolones with poor activity in some developing countries, has been responsible for the rapid development of bacterial resistance to these agents. ${ }^{1}$

Urinary tract infection (UTI) is second on ranking of the most common infection in community practice and has been diagnosed most commonly in outpatients as well as hospitalized. ${ }^{4}$ It is the most common diseases encountered in medical practices. However, its impact and frequency fluctuate in different demographic and geographic populations. 5,6 An estimated $10 \%$ of the population is diagnosed with UTIs annually. ${ }^{7}$ It also constitutes the most common nosocomial infection in many hospitals, plus its occurrence is about 2-3 cases per 100 hospital admissions and accounts for approximately $35 \%-40 \%$ of all hospital-acquired infections reported to the National Nosocomial Infection Surveillance (NNIS) system as in U.S.A. ${ }^{8,9}$ An estimated $20-30 \%$ of women are expected to have UTI after childhood 6 . UTIs result in approximately 8 million physician visits and more than 100,000 hospital admissions per year in the United States. ${ }^{10,11}$

UTI may involve the lower urinary tract or both the upper and lower tract. In case of lower UTI it is referred to as cystitis, the characteristics of which include a syndrome involving dysuria, frequency, urgency and occasionally suprapubic tenderness. However, the presence of symptoms of lower tract without upper tract symptoms does not necessarily exclude upper tract infection, as well ${ }^{3}$. In female patients uncomplicated cystitis is most common, whereas in male patients complicated UTIs are more common, while the proportion of re-infections and relapses in UTIs amounts to about $35-70 \% .12$

Escherichia coli is stated to be the dominant bacterial pathogen of community acquired UTIs. Most cases of UTIs are due to Escherichia coli and Staphylococci spp. while other agents like
Proteus spp. and Klebsiella pneumoniae account for many of the other infections also. ${ }^{8}$ Gram-positive bacteria, such as Staphylococci saprophyticus and Enterococcus spp. can result in UTIs in certain patients. For example, $S$. saprophyticus is a common pathogen in young, sexually active women and causes approximately $10 \%$ of UTIs in them. ${ }^{13}$

The causative agents of UTI and their antibiotic susceptibility have been changing over the years, both in community and hospital-acquired infections which is of great concern. ${ }^{14,15}$ Hence knowledge on the present pattern of susceptibility of antibiotics is important for appropriate therapy. UTIs are often treated with different broad-spectrum antibiotics, even if one with a narrow spectrum of activity may be appropriate because of concerns about infection with resistant organisms. ${ }^{16}$ Therefore extensive uses of antimicrobial agents have consistently resulted in the development of antibiotic resistance, which, in recent years, has become a main problem worldwide. 8,17 A study showed that though the frequency of resistance differed among laboratories, however, the trend towards increasing resistance was demonstrated in every participating laboratory. ${ }^{18}$

Knowledge of antimicrobial resistance patterns of the major pathogens linked with UTI is essential as a guide in selecting empirical antimicrobial therapy, because several authors have illustrated a relationship between the usage of antibiotics and emergence of resistance. ${ }^{19}$ The susceptibility pattern of community-acquired UTI pathogens has been acknowledged in a few studies. ${ }^{20}$ It is stated, that the elevated resistance in older age groups (mainly female) might consequence from increased cumulative lifetime exposure in the older patients. ${ }^{21}$ Data from the multivariate analysis point out that the effect of age on fluoroquinolone resistance is strongly influenced by gender, maybe due to the different physiological nature of UTI in males and females. The study aims to investigate the prevalence and susceptibility pattern of pathogens, causing urinary tract infections (UTIs), to antibiotics commonly used in routine medication.

\section{MATERIALS AND METHODS}

\section{Collection, Storage and Identification of samples}

A total of 100 isolates were collected from a diagnostic laboratory (MedPath Laboratories and diagnostic center, Karachi) over a period of 10 month. For the purpose of collecting the isolates, the 
respective laboratory was asked to provide only isolates considered significant by their usual criteria. For storage the isolates were sub cultured on to Nutrient Agar (Oxoid) slopes and stored for batched testing, the cultures were checked for purity by sub culturing on to Cysteine Lactose Electrolyte Deficient (CLED) agar (Oxoid) for Gramnegative isolates and blood agar (Oxoid) for Grampositive isolates. Whereas in the case of mixed cultures, no more than two bacteria (those with the two highest counts) were identified and then later on processed. The significant pathogens were identified by performing Gram staining and by determining their morphological features and via standard biochemical procedures. ${ }^{22}$

\section{Antibiotic Sensitivity Test}

After re-identification, the Minimum inhibitory concentration (MIC) for seven different antimicrobials (Oxoid) (Table-1) were determined by the agar dilution method, as described in the National Committee for Clinical Laboratory Standards (NCCLS ; presently called as Clinical Laboratory Standard Institute (CLSI) guidelines, on Mueller-Hinton agar, ${ }^{24}$ and interpreted according to CLSI procedures and interpretive guidelines. ${ }^{13}$ Antimicrobial susceptibility testing was performed using the disk diffusion method as described by the CLSI. ${ }^{25}$

\section{RESULTS}

Out of 100 isolates, 89 were found to be Gram negative and the rest were Gram positive. The following bacteria were classified as uropathogens; E. coli, Klebsiella spp., other Enterobacter spp., Staphylococci spp. and Pseudomonas spp. Which means $60 \%$ of isolates belonged to the Enterobacteriaceae, while in many cases like Hryniewicz K et.al more than $90 \%$ of isolates were found to be of this group. ${ }^{24}$ The Enterobacteriaceae, were the most frequent pathogens detected, causing $84.3 \%$ of the UTIs. The overall frequency of infection and the pathogens involved were broadly as expected and confirm that E. coli is the most common pathogen. A very low incidence of community infections caused by Gram-positive bacteria was also observed as by, as only $20 \%$ in this study. Identified microorganisms were accounted by E. coli (43\%), Klebsiella spp. (13\%). The occurrence frequency of different pathogens and their susceptibility to different antibiotics used are shown in Figure 1.

\section{DISCUSSION}

This study like many others indicated that E. coli is still the most common (43\%) cause of UTI. This corresponds with the data obtained by other investigators. ${ }^{13}$ An almost similar results were shown by a study conducted at Aga Khan University (AKU) Pakistan in 1994 where, E. coli was found most frequently (61.4\%) followed by Klebsiella spp. (17.1\%). Some have shown, however, that the percentage of $E$. coli is slowly declining, being replaced by other members of the Enterobacteriaceae and Enterococci spp. ${ }^{24}$ In general $E$. coli was susceptible to many drugs $(81.39 \%)$; this data is similar to those obtained in other countries indicating that $E$. coli is still susceptible to many antimicrobial agents. ${ }^{24,}$ 26-27 However, a high percentage of multi-resistant strains were found amongst other Enterobacteriaceae. In our country (Pakistan), multidrug resistant isolates of $E$. coli are much higher to $3^{\text {rd }}$ generation cephalosporin and quinolones. Findings from routine testing by medical microbiological laboratories also show the growing resistance to several antibiotics in $E$. coli from urinary tract isolates. ${ }^{18}$ Though the incidence of resistance differed among laboratories, perhaps owing to different testing methods. However, the trend towards increasing resistance is demonstrated in every participating laboratory. 11

Albeit, Pseudomonas species are fairly uncommon to be sensitive to drugs, as they have many ways of resistance, yet in this study they were found to be susceptible to Imipenem (85\%) followed by being sensitive to gentamicin and ofloxacin, $81 \%$ and $71.4 \%$ respectively. However on the other hand, showing its real devil shadow, giving resistance to the rest of antibiotics, indicating a worrying concern.

Staphylococci are said to be the champion in resistance, and almost the same happened in this study where off 11 Staphylococci isolates $95 \%$ proved to be resistant to one or more antibiotics used. Among antibiotics the most resistance was proved to cefixime and ofloxacin where $100 \%$ resistance was shown and even no intermediate range were found to be present, the next antibiotic to be resisted mostly was gentamicin where off only 1 isolate was sensitive. These were followed by amoxicillin. The antimicrobial agent to which Staphylococci spp. showed most 


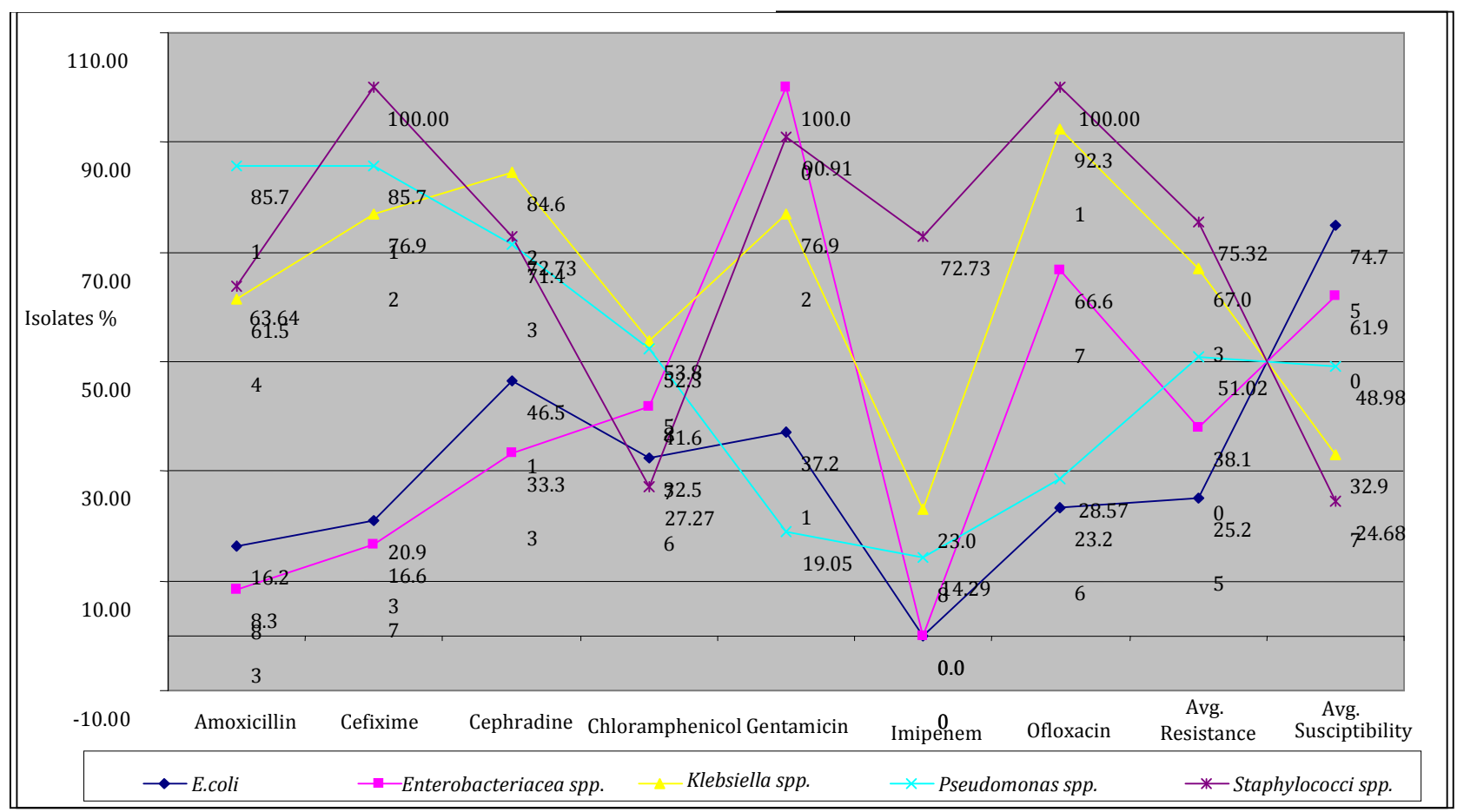

Figure 1. Resistance pattern of uropathogens

sensitivity was chloramphenicol, where $72.72 \%$ proved to be sensitive, almost the same conditions, as followed by the other four isolates.

Klebsiella spp. are the third major population to be the etiological agent of most of urinary tract infections, therefore must be considered for their sensitivity tests. This specie showed resistance to most of the agents, in different ratios (Fig. 1). It showed $92 \%$ resistant to ofloxacin, the most of all, following cefixime and gentamicin with resistance being $83.3 \%$. The other agent to which it showed it resistance included amoxicillin and chloramphenicol. However, it proved to be sensitive to imipenem with a ratio of $75 \%$ followed by $50 \%$ by chloramphenicol. In some of agents like amoxicillin it showed no sensitivity but with resistance some of the isolates showed intermediate as $41 \%$ in this case and $25 \%$ in Gentamycin.

Amoxicillin/ clavulanic acid combination is fairly active against organism sensitive to Amoxicillin for example Staphylococci species and also clavulinic acid has been shown to act synergistically with penicillin in vitro against clinical isolates of Blactamase producing strains of Staphylococci, however in this study the Staphylococci were found to be resistant to this combination $(7 / 11 \%)$. These observations connote to the partial failure of the used of this combination against Staphylococci. Albeit, the presence of some vulnerable isolates
" $4 / 11 \%$ still suggest the utilization of this combination. The efficacy of Augmentin against $E$. coli is quite effective, as $36 / 41 \%$ of isolates proved susceptible to this combination and the similar results were found in a no research works.

Cefixime is the extended spectrum, classified as $3^{\text {rd }}$ generation broad spectrum Cephalosporins. The oral cephalosporin is more stable than narrow spectrum against Gram-negative and other species. The sensitivity of Enterobacter spp. and E. coli of this scripture are in submission, however it was found to be not as effective in case of other species as Pseudomonas, Klebsiella spp. and Staphylococci, as given in figure 1.

Data presented in this study also indicate that antibiotics commonly used in UTIs are nevertheless still effective, but species distribution and their susceptibility to antibiotics are changing in general all around the world. Hence it requires regular monitoring in order to make reliable information available for optimal empirical therapy for patients with UTIs.

To prevent a further increase of resistance, it is strictly recommended that antibiotics should be used if urinary tract isolates are susceptible only to these antibiotics or as a second-choice drug for patients in whom treatment with the antibiotic of first choice has failed. Although prudent use of these 
antibiotics may help to reduce the prevalence of antibiotic-resistant uropathogens, elimination will be hard to achieve. Therefore, we believe that we can reduce the tide but we will still have to deal with the resistance problem in the future. ${ }^{7}$ Because bacterial resistance leads not only to longer and more costly hospital stays but also to increased morbidity and death rates ${ }^{26}$ therefore amongst the numerous recommendations to combat resistant bacteria is the necessity for national and international surveillance programmes to monitor the level of antimicrobial resistance. Several programmes have been investigated which have looked at pathogens from a variety of common infections. These include the WHO Antimicrobial Resistance Monitoring Programme, the European Antimicrobial Resistance Surveillance System (EARSS), the Hospitals in Europe Link for Infection Control through Surveillance (HELICS), the European Study Group on Nosocomial Infections, the SENTRY Antimicrobial Surveillance Program has been monitoring antimicrobial resistance worldwide among selected types of infection since January $1997^{9}$ and the Alexander Project on pathogens in lower respiratory tract infections. ${ }^{22}$ Similarly the establishment of worldwide antimicrobial resistance surveillance systems seems to be an important step in detecting the emergence of resistance patterns. ${ }^{9}$

\section{CONCLUSIONS}

In the conclusion it can be stated that the though resistance UTI pathogens has been increasing rapidly, however with advent of new antibiotics it can be overcome. In this context according to our study the use of ofloxacin is the drug of choice for the treatment of UTI caused by either Gram positive or Gram-negative pathogens. Whereas Gentamicin is additionally recommended for $E$. coli.

\section{ACKNOWLEDGEMENTS}

We are indebted to Med Path Laboratories and Diagnostic center Karachi, for the provision of the UTI isolates and their primary identification information.

CONFLICT OF INTERESTS: None to declare.

FINANCIAL INTEREST: None to declare.

\section{REFERENCES}

1. Ruiz J. Mechanisms of resistance to quinolones: target alterations, decreased accumulation and DNA gyrase protection. J Antimicrob Chemother 2003;51:1109-1117. Epub 2003/04/17.

2. Acar JF, Goldstein FW. Trends in bacterial resistance to fluoroquinolones. Clin Infect Dis 1997;24 Suppl 1:S6773. Epub 1997/01/01.

3. Davis R, Markham A, Balfour JA. Ciprofloxacin. An updated review of its pharmacology, therapeutic efficacy and tolerability. Drugs 1996;51:1019-1074. Epub 1996/06/01.

4. Gonzalez CM, Schaeffer AJ. Treatment of urinary tract infection: what's old, what's new, and what works. World J Urol 1999;17:372-82. Epub 2000/02/02.

5. Dimitrov TS, Udo EE, Emara M, Awni F, Passadilla R. Etiology and antibiotic susceptibility patterns of community-acquired urinary tract infections in a Kuwait hospital. Med Princ Pract 2004;13:334-9. Epub 2004/10/07.

6. Kunin CM. Urinary tract infections in females. Clin Infect Dis 1994;18:1-10; quiz 1-2. Epub 1994/01/01.

7. Fluit AC, Schmitz FJ. Bacterial resistance in urinary tract infections: how to stem the tide. Expert Opin Pharmacother 2001;2:813-8. Epub 2001/05/05.

8. Barnett BJ, Stephens DS. Urinary tract infection: an overview. Am J Med Sci 1997;314(4):245-9. Epub $1997 / 10 / 23$.

9. Gales AC, Jones RN, Gordon KA, Sader HS, Wilke WW, Beach ML, et al. Activity and spectrum of 22 antimicrobial agents tested against urinary tract infection pathogens in hospitalized patients in Latin America: report from the second year of the SENTRY antimicrobial surveillance program (1998). J Antimicrob Chemother 2000;45:295303. Epub 2000/03/07.

10. Sahm DF, Thornsberry C, Mayfield DC, Jones ME, Karlowsky JA. Multidrug-resistant urinary tract isolates of Escherichia coli: prevalence and patient demographics in the United States in 2000. Antimicrob Agents Chemother 2001;45(5):1402-6. Epub 2001/04/17.

11. Warren JW, Abrutyn E, Hebel JR, Johnson JR, Schaeffer AJ, Stamm WE. Guidelines for antimicrobial treatment of uncomplicated acute bacterial cystitis and acute pyelonephritis in women. Infectious Diseases Society of America (IDSA). Clin Infect Dis 1999;29:745-58. Epub 1999/12/10.

12. Wolfhagen MJ, Hoepelman IM, de Melker RA, Verhoef J. [Diagnosis of uncomplicated urinary tract infections: complicated?]. Ned Tijdschr Geneeskd 1988;132:390-4. Epub 1988/02/21. Diagnostiek van ongecompliceerde urineweginfecties: gecompliceerd?

13. Latham RH, Running K, Stamm WE. Urinary tract infections in young adult women caused by Staphylococcus saprophyticus. J Am Med Assoc 1983;250:3063-3066.

14. Neu HC. Urinary tract infections. Am J Med 1992;92:63S-70S. Epub 1992/04/06.

15. Schaeffer AJ. Urinary tract infections in the elderly. Eur Urol 1991;19 Suppl 1:2-6. Epub 1991/01/01. 
16. Schaeffer AJ. The expanding role of fluoroquinolones. Am J Med 2002;113 Suppl 1A:45S-54S. Epub 2002/07/13. 17. Sirot DL, Goldstein FW, Soussy CJ, Courtieu AL, Husson MO, Lemozy J, et al. Resistance to cefotaxime and seven other beta-lactams in members of the family Enterobacteriaceae: a 3-year survey in France. Antimicrob Agents Chemother 1992;36:1677-81. Epub 1992/08/01.

18. Goettsch W, van Pelt W, Nagelkerke N, Hendrix MG, Buiting AG, Petit PL, et al. Increasing resistance to fluoroquinolones in escherichia coli from urinary tract infections in the netherlands. J Antimicrob Chemother 2000;46:223-8. Epub 2000/08/10.

19. Ena J, Lopez-Perezagua MM, Martinez-Peinado C, CiaBarrio MA, Ruiz-Lopez I. Emergence of ciprofloxacin resistance in Escherichia coli isolates after widespread use of fluoroquinolones. Diag Microbiol Infect Dis 1998;30:103-7. Epub 1998/04/29.

20. Weber G, Riesenberg K, Schlaeffer F, Peled N, Borer A, Yagupsky $P$. Changing trends in frequency and antimicrobial resistance of urinary pathogens in outpatient clinics and a hospital in Southern Israel, 19911995. Eur J Clin Microbiol Infect Dis 1997;16:834-8. Epub 1998/02/03.

21. Warren JW. Clinical presentations and epidemiology of urinary tract infections, In Urinary Tract Infections, Molecular Pathogenesis and Clinical Management, (Mobley, H. L. \& Warren, J. W., Eds). 1996:3-27. ASM Press, Washington, DC.

22. Kahlmeter G. An international survey of the antimicrobial susceptibility of pathogens from uncomplicated urinary tract infections: the ECO.SENS Project. J Antimicrob Chemother 2003;51:69-76. Epub 2002/12/21.

23. Felmingham D, Robbins MJ, Tesfaslasie Y, Harding I, Shrimpton S, Gruneberg RN. Antimicrobial susceptibility of community-acquired lower respiratory tract bacterial pathogens isolated in the UK during the 1995-1996 cold season. J Antimicrob Chemother 1998;41:411-5. Epub 1998/05/13.

24. Hryniewicz K, Szczypa K, Sulikowska A, Jankowski K, Betlejewska K, Hryniewicz W. Antibiotic susceptibility of bacterial strains isolated from urinary tract infections in Poland. J Antimicrob Chemother 2001;47:773-80. Epub 2001/06/05.

25. Wayne. Methods for Disk Susceptibility Tests for Bacteria That Grow Aerobically. National Committee for Clinical Laboratory Standards. 2000 (NCCLS Documant M2-A7).

26. Stamm WE, Hooton TM. Management of urinary tract infections in adults. N Engl J Med 1993;329:1328-34. Epub $1993 / 10 / 28$.

27. Ferry S, Burman LG, Holm SE. Clinical and bacteriological effects of therapy of urinary tract infection in primary health care: relation to in vitro sensitivity testing. Scand J Infect Dis 1988;20:535-44. Epub 1988/01/01.

\section{Citing this article}

Najmul H, Tanveer A. Changing pattern of resistant pathogens causing urinary tract infections: a resistance trend of UTI pathogens in Karachi. Int J Infect Microbiol 2013;2(3):104-110. 